\title{
Increased EEG gamma band activity in Alzheimer's disease and mild cognitive impairment
}

\author{
J. A. van Deursen · E. F. P. M. Vuurman • \\ F. R. J. Verhey • V. H. J. M. van Kranen-Mastenbroek • \\ W. J. Riedel
}

Received: 29 April 2008/Accepted: 15 June 2008/Published online: 8 July 2008

(C) The Author(s) 2008

\begin{abstract}
High frequency $(30-70 \mathrm{~Hz})$ gamma band oscillations in the human electro-encephalogram (EEG) are thought to reflect perceptual and cognitive processes. It is therefore interesting to study these measures in cognitive impairment and dementia. To evaluate gamma band oscillations as a diagnostic biomarker in Alzheimer's disease $(\mathrm{AD})$ and mild cognitive impairment (MCI), 15 psychoactive drug naïve $\mathrm{AD}$ patients, $20 \mathrm{MCI}$ patients and 20 healthy controls participated in this study. Gamma band power (GBP) was measured in four conditions viz. resting state, music listening, story listening and visual stimulation. To evaluate test-retest reliability (TRR), subjects underwent a similar assessment one week after the first. The overall TRR was high. Elevated GBP was observed in $\mathrm{AD}$ when compared to $\mathrm{MCI}$ and control subjects in all conditions. The results suggest that elevated GBP is a reproducible and sensitive measure for cognitive dysfunction in $\mathrm{AD}$ in comparison with $\mathrm{MCI}$ and controls.
\end{abstract}

J. A. van Deursen · E. F. P. M. Vuurman $(\bowtie)$ · F. R. J. Verhey

Department of Psychiatry and Neuropsychology,

Faculty of Health, Medicine and Life Sciences,

Maastricht University, P.O. Box 616,

6200 Maastricht, The Netherlands

e-mail: E.Vuurman@NP.unimaas.nl

J. A. van Deursen - E. F. P. M. Vuurman - W. J. Riedel Department of Neuropsychology and Psychopharmacology, Faculty of Psychology, Maastricht University,

Maastricht, The Netherlands

V. H. J. M. van Kranen-Mastenbroek

Department of Clinical Neurophysiology, Maastricht University

Hospital, Maastricht, The Netherlands
Keywords EEG · Induced gamma band .

Alzheimer's disease $\cdot$ Mild cognitive impairment .

Sensitivity $\cdot$ Reliability

\section{Introduction}

Alzheimer's disease (AD) is a progressive neurodegenerative disorder with clinical manifestations of loss of cognitive functions and impaired daily life activities.

The neurophysiological basis for the cognitive and behavioural dysfunction in $\mathrm{AD}$ is not completely understood but involves changes in a variety of neural substrates throughout the brain. Frequency analysis of ongoing (background) electroencephalography (EEG) provides a good indication about the neurological integrity of the central nervous system (CNS) and has as such often been used in $\mathrm{AD}$ studies. In clinical studies the frequency spectrum of EEG is divided in four frequency bands; delta $(<4 \mathrm{~Hz})$, theta $(4-8 \mathrm{~Hz})$, alpha $(8-13 \mathrm{~Hz})$ and beta $(13-$ $30 \mathrm{~Hz}$ ). The EEG in AD is characterised by an increase of power in the lower frequencies and a decrease in the power of the higher frequencies (Huang et al. 2000; Jelic et al. 1996; Jeong 2004). The decline in power of the higher frequencies is a consistent finding in $\mathrm{AD}$ that correlates with cognitive performance, but nevertheless has no etiological specificity for AD. It is especially difficult to discriminate $\mathrm{AD}$ from vascular dementia $(\mathrm{VaD})$, based on clinical EEG (Jeong 2004).

A basic question in functional brain research is how the manifold of serial and parallel neuronal activations needed to process basic stimuli are integrated and bound together. The precise timing and integration of neural activation is crucial for successful information processing in the brain. Several studies have suggested an important role for very 
high frequency or gamma band activity $(30-100 \mathrm{~Hz})$ in the timing and integration process of neuronal networks in the brain (Singer and Gray 1995; Basar et al. 2001; Kaiser and Lutzenberger 2003). Gamma band oscillations can thus be considered as neural activity that is generated to synchronize sub-processes in neural networks that are involved in specific information processing (Kaiser and Lutzenberger 2003, 2005). High frequency discharges and synchronization seem to be responses to stimuli, as a mechanism for the integration of different features of a stimulus to a whole (Tallon-Baudry and Bertrand 1999). As reviewed by (Tallon-Baudry and Bertrand 1999) there are three ways to describe gamma band activity: (1) the gamma band evoked response which is characterized by EEG activity that is phase-locked to the onset of a certain stimulus and is therefore directly related to it; (2) the $40 \mathrm{~Hz}$ steady-state response (SSR) which is a sinusoidal response at the driving stimulus frequency and is elicited by simple visual, auditory or somatosensory stimuli. This SSR can be interpreted as a natural resonance frequency of the brain and is related to primary sensory processing (Galambos et al. 1981); (3) induced gamma band oscillations which result from continuous sensory stimulation and are not time or phase locked to a specific stimulus but elevated during meaningful information processing. Taken together, gamma band activity and synchronicity play an important role in both primary stimulus processing as well as higher information processing.

In Alzheimer's disease several studies have reported decreased synchronization of the gamma band (Koenig et al. 2005; Stam et al. 2002, 2003) but none of these human studies have focused on induced gamma band power (GBP). EEG power expresses the magnitude and intensity of the local EEG signal and is calculated by the squared amplitude of the oscillations. Synchronization is a numerical property of the association between two or more different EEG electrodes requiring groups of neurons distant from each other firing in the same phase and/or frequency. In comparison to synchronization, EEG power could be a simpler, faster and more straightforward measure to examine changes in gamma band oscillations in patient groups. Furthermore, most studies on gamma band activity in $\mathrm{AD}$ have been assessed in patients using psychoactive medication. To rule out these potential medication effects as confounding with diagnosis, only patients naïve for psychoactive drugs should be included in studies comparing gamma band activity between patients and controls.

The current study focuses on induced gamma band power and has two major objectives. The primary objective is to evaluate the diagnostic sensitivity of induced GBP in discriminating psychoactive drug naïve $\mathrm{AD}$ patients from MCI patients and healthy controls. The secondary objective is to evaluate the reliability and stability of induced GBP in cognitively impaired patients. The design for the first objective is based on a study by Fitzgibbon et al. (2004), which showed gamma band augmentation in a variety of relatively simple cognitive tasks in healthy volunteers. Music listening, story listening and movie watching are highly over-learned which makes them particularly suitable to be applied in cognitively impaired patients.

In the current study we determined whether differences in GBP, induced by the cognitive task load of these paradigms, could discriminate AD patients, MCI patients and healthy controls. Since information processing is disturbed in AD and MCI we hypothesised that induced GBP would be lower in $\mathrm{AD}$ subjects than in MCI and controls subjects in all tasks conditions. Furthermore, it was to be expected that GBP in MCI was lower than in the control and higher than in the AD group.

\section{Materials and methods}

Subjects

The study involved three different groups of subjects:

1. Fifteen psychoactive drug naïve patients with a diagnosis of probable AD according to the NINCDSADRDA criteria (McKhann et al. 1984) were included. Standard blood workup and neuroimaging (CT or MRI) were carried out, and the diagnosis was supported by abnormal performance on neuropsychological testing.

2. Twenty psychoactive drug naïve patients with a diagnosis of MCI according to the Petersen criteria were included (Petersen et al. 2001). MCI subjects also received standard blood workup, neuroimaging and neuropsychological testing. The diagnosis AD or MCI was made in a weekly consensus meeting of different specialists.

3. Twenty healthy control subjects were recruited from the Maastricht Aging Study (MAAS), a longitudinal study of the determinants of healthy cognitive aging (van Boxtel et al. 1998). The control subjects were not using any psychoactive medications. The medical history was screened by a medical health questionnaire. Main exclusion criteria were a history of stroke, head trauma, or any other neurological or psychiatric disorders. Additional exclusion criteria were severe cardiovascular disease, a Hachinski ischemic scale (HIS) (Hachinski et al. 1974; Rosen et al. 1980) higher than three or a history of substance abuse and/or other serious system diseases (e.g. malignancy, uncontrolled 
hypertension, neuropathy or seizure disorders). All AD and MCI patients were recruited at the Memory Clinic of University Hospital Maastricht and were judged competent to give consent by their treating physicians. This study was carried out in accordance with the declaration of Helsinki. All participants gave written informed consent prior to the study, for AD patients a family member also signed the consent form. The local Medical Ethics Committee of University Hospital Maastricht approved the study.

\section{Experimental procedure}

All subjects took part in two identical recording sessions, spaced apart one week. Prior to EEG acquisition, the cognitive subscale of the Alzheimer's disease assessment scale (ADAS-cog) (Rosen et al. 1984; Verhey et al. 2004) was administered, on both occasions. The Dutch version of the National Adult Reading Test (NART) (Schmand et al. 1991) was administered to estimate pre-morbid intelligence. The three outcome measures; music listening, story listening and movie watching were part of a larger study, which further included: auditory $40-\mathrm{Hz}$ steady state stimulation, contingent negative variation (CNV), a checkerboard reversal task and an event related potentials (ERP) paradigm. Continuous EEG was recorded while subjects performed the music listening task, story listening task and movie watching task. Headphones were used to present the auditory stimuli. To evaluate test-retest reliability (TRR) of the acquired data, all subjects were tested twice with an interval of at least one week.

\section{EEG acquisition}

A commercially available Nuamps ${ }^{\circledR}$ EEG acquisition system was used to record the EEG's. Electrodes $(\mathrm{AgCl})$ were positioned following the 10-20 system on a 32-channel electrode cap, using 19 Medcat $^{\circledR}$ electrodes. A low pass filter of $100 \mathrm{~Hz}$ and a high pass filter of $0.1 \mathrm{~Hz}$ were applied. The sample frequency was $512 \mathrm{~Hz}$ and analoguedigital conversion was 20 bit. Electrode impedance was kept below $5 \mathrm{k} \Omega$. A reference electrode was placed on the right ear lobe. To control for possible vertical eye movements, an electro-oculogram (EOG) electrode was placed $1 \mathrm{~cm}$ under the midline of the right eye. A ground electrode was placed on the forehead, at Fpz. EEG's were recorded in a magnetically shielded and sound attenuated room. The visual stimuli were presented on a flat, LCD screen located outside the shielded room at eye height. Subjects were able to see the screen through magnetically shielded glass. Neuroscan ${ }^{\circledR} 4.3$ software was used for EEG recording and analyses.
EEG gamma band paradigms

During the resting state measurement, subjects were instructed to keep their eyes open and fixate at a white crosshair located on a black screen for $90 \mathrm{~s}$.

During the music listening task, subjects were instructed to listen for $90 \mathrm{~s}$ to broad-spectrum acoustical music. While listening to the stimuli subjects fixated on a white crosshair on a black screen and kept their eyes open. The music was a segment from Enya's "From where I am".

During the story listening task subjects listened for tree minutes to a fragment of a Dutch spoken documentary (Fly Away 2, fragment Australia). While listening to the stimuli subjects had to fixate on a white crosshair on a black screen with their eyes open. The fragment was spoken monotonously, and pauses were not longer than $5 \mathrm{~s}$. Subjects were instructed to listen carefully to the spoken text.

In the visual task a series of moving abstract objects were presented for $180 \mathrm{~s}$. The objects were coloured and moved at variable speed. This was a fragment of recorded Windows ${ }^{\circledR}$ screensaver. Subjects were instructed to monitor the movements carefully.

\section{EEG analysis}

EEG analysis was performed off-line. The continuous EEG files were cut in epochs of $1 \mathrm{~s}$. Epochs containing eyemovement, electromyographic activity and head motion artefacts were excluded from analysis. The average number of uncontaminated epochs in resting state condition was 62 (AD: 55, MCI: 63, Control: 67). In the music listening task the average number of uncontaminated epochs was 67 (AD: 60, MCI: 67, Control: 74). In the story listening task the average number of uncontaminated epochs was 153 (AD: 145, MCI: 161, Control: 153). In the visual task the average number of uncontaminated epochs was 85 (AD: 83, MCI: 89, Control: 84.) The uncontaminated epochs were transformed from the temporal domain to the frequency domain using fast-Fourier transformation $(1 \mathrm{~Hz}$ resolution, 512 point block-size, Hanning window 1$100 \mathrm{~Hz}$ ). The gamma band was defined as the frequency between 30 and $100 \mathrm{~Hz}$. A notch filter of $50 \mathrm{~Hz}$ was applied to omit contamination of the electrical light network in the room. The frequency epochs were averaged and absolute GBP was calculated for every task, subject and electrode.

\section{EMG analysis}

In order to ensure that the results are related to EEG differences and not to electromyographic (EMG) contamination (Whitham et al. 2007), an extra analysis was performed on those electrodes that previously showed GBP 
differences between the groups. The procedure involved low-pass amplitude filtering and visual inspection of the EEG epochs under guidance of a clinical EEG expert. Obvious and suspect epochs for EMG contamination were omitted from further analysis. The number of "clean" epochs was in resting state 53 (AD: 43, MCI: 57, Control: 61), in music listening 56 (AD: 52, MCI: 64, Control: 52), in story listening 131 (AD: 128, MCI: 135) and in the visual task 68 (AD: 71, MCI: 68, Control: 64). These "corrected" EEG epochs were further processed and analysed in the same manner as the original data. A paired sample $t$-test was used to examine differences between the EMG corrected an uncorrected data.

\section{Statistics}

Statistical analysis was done with SPPS for Windows (version 11.5). Significance levels associated with the differences in GBP between the $\mathrm{AD}, \mathrm{MCI}$ and control groups were calculated for each electrode using ANOVA for repeated measures. Electrode positions were used as a within subject variable. Since GBP was calculated in two identical sessions, the mean of these two sessions was used to calculate between group differences. Post-Hoc Bonferonni correction for multiple comparisons was used. For the ANOVA for repeated measures analysis, the mean EEG power values of the two sessions were calculated for each subject and each electrode.

To examine test-retest reliability of GBP within the different paradigms, a paired $T$-test was used to evaluate potential differences between the two sessions for the Fz, $\mathrm{Cz}$ and $\mathrm{Pz}$ electrode. Furthermore, Pearson's correlation coefficient was calculated for each task at $\mathrm{Fz}, \mathrm{Cz}$ and $\mathrm{Pz}$. These analyses were done for each patient group separately.

To examine the relation between GBP and cognitive performance measured by ADAS-cog, Pearson's correlation coefficient was calculated between GBP and ADAS$\operatorname{cog}$.

To test whether the stimuli induced increased GBP, these conditions were compared to resting state using ANOVA. The differences between the groups in changes in GBP in task condition compared to resting state were examined by calculating the ratio between task and resting state. Differences in this ratio between groups were analysed using ANOVA for repeated measures.

\section{Results}

Subject's demographics

Fifteen AD patients (11 male, 4 female) with a mean age (SD) of 75.2 years (6.9) and a mean (SD) MMSE score of
20.8 (2.7, range 17-26), 20 (12 male, 8 female) MCI patients with a mean (SD) age of 70.6 (7.2) and mean (SD) MMSE of 26.3 (1.6, range 23-29) and, 20 (12 male, 8 female) healthy control subjects with a mean (SD) age of 69.5 (6.1) and mean (SD) MMSE of 29.3 (0.8, range 2830 ) completed the study. There was no statistical difference in age between the groups $\left(F_{2,52}=2.2, P=0.14\right)$.

Neuropsychological tests

Average ADAS-Cog scores were 19.6 (SD: $5.9 ; n 15$ ) in the AD group, 10.8 (SD: $4.5 ; n$ 20) in the MCI group and 5.8 (SD: $2.6 ; n$ 20) in the control group. These scores showed a significant difference between the groups in the expected direction $\left(F_{2,52}=84.8, P<0.001\right)$. Results from the NART showed that the mean estimated pre-morbid intelligence was $96.9\left(F_{2,52}=2.3, P=1.03\right)$ and did not differ between the groups.

\section{Test-retest reliability (TRR)}

The paired samples $T$-test revealed a significant difference between the sessions in the resting state condition in the $\mathrm{AD}$ group at electrode $\mathrm{Cz}$ and in the control group at $\mathrm{Cz}$ and $\mathrm{Pz}$ electrode (see Table 1). In the other task conditions there were no significant differences between the two sessions.

Pearson's correlation coefficient between the two sessions was very high in resting state, music listening, and story listening for all the groups (see Table 1). The visual task showed a lower correlation between the sessions for all the groups.

\section{Induced gamma band paradigms}

Figure 1 summarizes the results of GBP in the four conditions.

In resting state, GLM showed differences between the group for $\mathrm{C} 3\left(F_{2,52}=3.83, P=0.037\right), \mathrm{P} 3\left(F_{2,52}=4.29\right.$, $P=0.032), \mathrm{Pz}\left(F_{2,52}=5.26, P=0.009\right), \mathrm{P} 4\left(F_{2,52}=\right.$ $4.51, \quad P=0.039), \quad$ T6 $\left(F_{2,52}=4.10, \quad P=0.020\right), \quad$ O1 $\left(F_{2,52}=3.42, \quad P=0.044\right) \quad$ and $\quad \mathrm{O} 2 \quad\left(F_{2,52}=6.33\right.$, $P=0.006)$. Bonferonni correction for multiple comparisons showed that GBP was higher in the AD group compared to the $\mathrm{MCI}$ at $\mathrm{C} 3$ and $\mathrm{O} 2$ and control group at $\mathrm{C} 3$, $\mathrm{P} 3, \mathrm{Pz}, \mathrm{P} 4, \mathrm{~T} 6, \mathrm{O} 1$ and $\mathrm{O} 2(P<0.05)$. There was no difference between the MCI and control group.

In the music listening task, GLM showed differences between the groups for T5 $\left(F_{2,52}=5.3, P=0.006\right)$, T6 $\left(F_{2,52}=5.2, P=0.012\right), \mathrm{P} 3\left(F_{2,52}=7.13, P=0.021\right)$, $\mathrm{Pz} \quad\left(F_{2,52}=7.02, \quad P=0.001\right), \quad \mathrm{O} 1 \quad\left(F_{2,52}=9.18\right.$, $P<0.004)$ and $\mathrm{O} 2\left(F_{2,52}=7.38, P<0.002\right)$. Bonferonni correction for multiple comparisons showed higher GBP in 
Table 1 Test-retest reliability of GBP

\begin{tabular}{|c|c|c|c|c|c|c|c|c|c|c|c|c|}
\hline & \multicolumn{4}{|c|}{ Alzheimer group } & \multicolumn{4}{|c|}{ MCI group } & \multicolumn{4}{|c|}{ Control group } \\
\hline & $\begin{array}{l}\text { Mean } \\
\mu V^{2} \text { s1 }\end{array}$ & $\begin{array}{l}\text { Mean } \\
\mu \mathrm{V}^{2} \text { s2 }\end{array}$ & $\begin{array}{l}\text { Sig. } \\
P\end{array}$ & $\begin{array}{l}\text { Pearson's } \\
R\end{array}$ & $\begin{array}{l}\text { Mean } \\
\mu V^{2} \text { s1 }\end{array}$ & $\begin{array}{l}\text { Mean } \\
\mu V^{2} \text { s2 }\end{array}$ & $\begin{array}{l}\text { Sig. } \\
P\end{array}$ & $\begin{array}{l}\text { Pearson's } \\
R\end{array}$ & $\begin{array}{l}\text { Mean } \\
\mu \mathrm{V}^{2} \mathrm{~s} 1\end{array}$ & $\begin{array}{l}\text { Mean } \\
\mu V^{2} \text { s2 }\end{array}$ & $\begin{array}{l}\text { Sig. } \\
P\end{array}$ & $\begin{array}{l}\text { Pearson's } \\
R\end{array}$ \\
\hline \multicolumn{13}{|c|}{ Resting state } \\
\hline $\mathrm{Fz}$ & 0.70 & 0.46 & 0.086 & 0.68 & 0.47 & 0.43 & 0.409 & 0.62 & 0.52 & 0.41 & 0.051 & 0.95 \\
\hline $\mathrm{Cz}$ & 0.65 & 0.48 & 0.037 & 0.89 & 0.48 & 0.43 & 0.284 & 0.71 & 0.49 & 0.41 & 0.036 & 0.96 \\
\hline $\mathrm{Pz}$ & 0.70 & 0.53 & 0.051 & 0.90 & 0.43 & 0.40 & 0.448 & 0.57 & 0.40 & 0.36 & 0.004 & 0.96 \\
\hline \multicolumn{13}{|c|}{ Music listening } \\
\hline $\mathrm{Fz}$ & 0.84 & 0.73 & 0.181 & 0.90 & 0.54 & 0.55 & 0.780 & 0.77 & 0.51 & 0.57 & 0.323 & 0.85 \\
\hline $\mathrm{Pz}$ & 0.86 & 0.75 & 0.136 & 0.92 & 0.51 & 0.54 & 0.394 & 0.84 & 0.48 & 0.53 & 0.409 & 0.81 \\
\hline $\mathrm{Cz}$ & 0.87 & 0.75 & 0.124 & 0.93 & 0.46 & 0.50 & 0.281 & 0.86 & 0.40 & 0.45 & 0.349 & 0.58 \\
\hline \multicolumn{13}{|c|}{ Story listening } \\
\hline $\mathrm{Fz}$ & 0.80 & 0.61 & 0.062 & 0.97 & 0.51 & 0.48 & 0.203 & 0.81 & 0.51 & 0.57 & 0.222 & 0.85 \\
\hline $\mathrm{Cz}$ & 0.79 & 0.61 & 0.091 & 0.98 & 0.50 & 0.46 & 0.154 & 0.90 & 0.49 & 0.52 & 0.436 & 0.84 \\
\hline $\mathrm{Pz}$ & 0.82 & 0.60 & 0.105 & 0.98 & 0.46 & 0.43 & 0.194 & 0.92 & 0.43 & 0.45 & 0.465 & 0.76 \\
\hline \multicolumn{13}{|c|}{ Visual task } \\
\hline $\mathrm{Fz}$ & 0.54 & 0.67 & 0.335 & 0.29 & 0.56 & 0.42 & 0.287 & 0.33 & 0.52 & 0.55 & 0.747 & 0.53 \\
\hline $\mathrm{Cz}$ & 0.52 & 0.66 & 0.373 & 0.33 & 0.51 & 0.41 & 0.379 & 0.35 & 0.47 & 0.50 & 0.671 & 0.56 \\
\hline $\mathrm{Pz}$ & 0.52 & 0.65 & 0.395 & 0.42 & 0.44 & 0.38 & 0.516 & 0.27 & 0.38 & 0.43 & 0.370 & 0.40 \\
\hline
\end{tabular}

The table presents the mean power in $\mu \mathrm{V}^{2}$ of the electrodes per session (i.e. $\mathrm{s} 1=$ session, $\mathrm{s} 2=$ session 2 ). The $P$-value represents the significance of the difference between the sessions. Pearson's $R$ represents the correlation coefficient between the two sessions

the AD group when compared to the MCI group at P3, O1, $\mathrm{O} 2$ and control group at T5, T6, P3, Pz, O1 and $\mathrm{O} 2$ $(P<0.05)$. There was no difference between MCI and control subjects.

In the story listening task, GLM showed a difference between the groups for $\mathrm{O} 2\left(F_{2,52}=7.23, P=0.003\right)$. Bonferonni correction for multiple comparisons showed significantly higher GBP in the AD group when compared to the control group. There was no difference between the MCI group and the control group. There was no significant difference between the groups at other electrodes.

In the visual task, ANOVA showed a difference between the groups for T5 $\left(F_{2,52}=4.13, P=0.029\right)$, O1 $\left(F_{2,52}=5.16, P=0.022\right)$ and $\mathrm{O} 2 \quad\left(F_{2,52}=6.82\right.$, $P=0.001)$. Bonferonni correction for multiple comparisons showed significantly higher GBP in AD compared to MCI at $\mathrm{O} 2$ electrode and control group at T5, O1 and $\mathrm{O} 2$ $(P<0.05)$. There was no significant difference between $\mathrm{MCI}$ and control group.

\section{Correlation between GBP and ADAS-Cog}

The correlations between GBP and ADAS-cog are presented in Table 2. The correlations were calculated for the electrodes that significantly separated the patient groups. The results showed a modest but significant correlation between GBP and ADAS-cog in all conditions and all analysed electrodes.
Differences between task and resting state condition

To examine if GBP is altered during task performance we compared GBP during task to resting state.

Figure 2 shows the electrodes with significantly increased GBP in task condition compared to resting state for each group separately together with the overall increase of GBP.

For music listening, GBP increased in the $\mathrm{AD}$ at $\mathrm{Fz}$ $\left(F_{1,1}=4.9, P=0.047\right), \mathrm{F} 4\left(F_{1,1}=5.6, P=0.029\right), \mathrm{F} 8$ $\left(F_{1,1}=5.9, P=0.031\right), \mathrm{Cz}\left(F_{1,1}=5.3, P=0.041\right)$ and T4 $\left(F_{1,1}=5.2, P=0.042\right)$. In the MCI group only $\mathrm{O} 2$ showed a significant increase of GBP $\left(F_{1,1}=5.1\right.$, $P=0.047)$ compared to resting state. In the control group music listening increased $\mathrm{GBP}$ in $\mathrm{F} 4 \quad\left(F_{1,1}=5.6\right.$, $P=0.029)$, C4 $\left(F_{1,1}=5.8, P=0.026\right), \mathrm{P} 4\left(F_{1,1}=5.4\right.$, $P=0.032)$ and T6 $\left(F_{1,1}=7.0, P=0.016\right)$ compared to resting state. When the increase of GBP in music listening was analysed for all subjects irrespective of patient group a significant difference was shown for Fz, F3, F4, F7, F8, Cz, T4, T5 and T6. The $F$ and $P$ values were in the same order of magnitude as when the groups were analysed separately. The percentages of increase in GBP due to music listening was $155 \%$ in $\mathrm{AD}, 28 \%$ in MCI and $40 \%$ in the control group. The AD group showed significantly higher increase of GBP due to music listening on T5 compared to MCI and controls. There were no other significant differences between the groups in GBP increase due to music listening. 

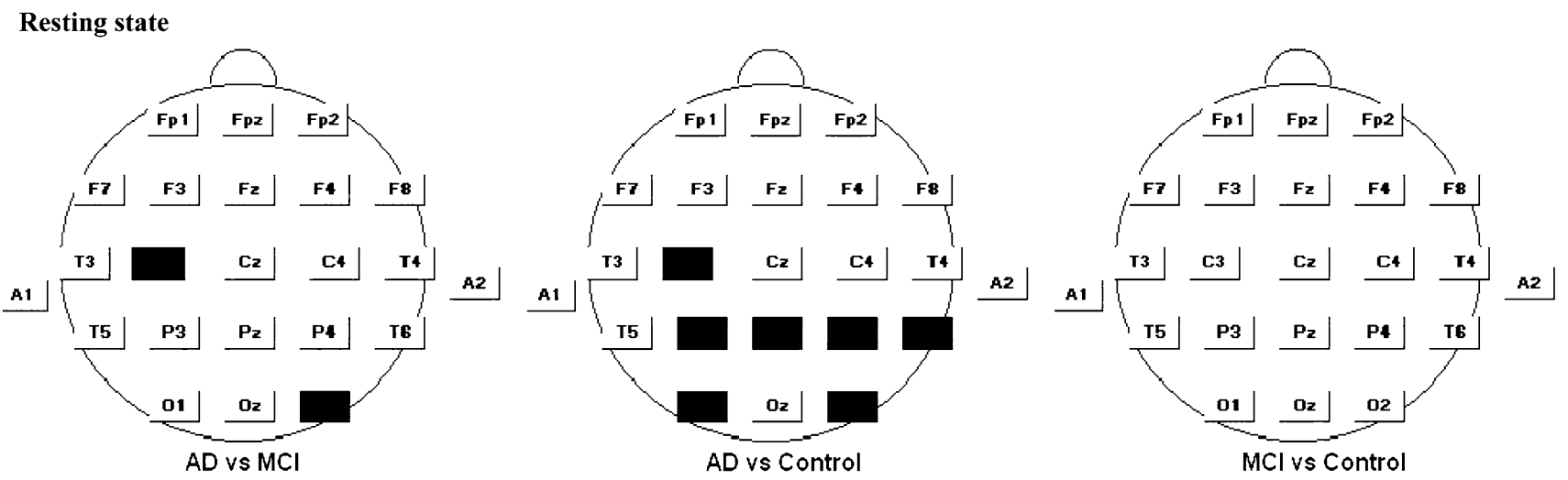

\section{Music listening task}
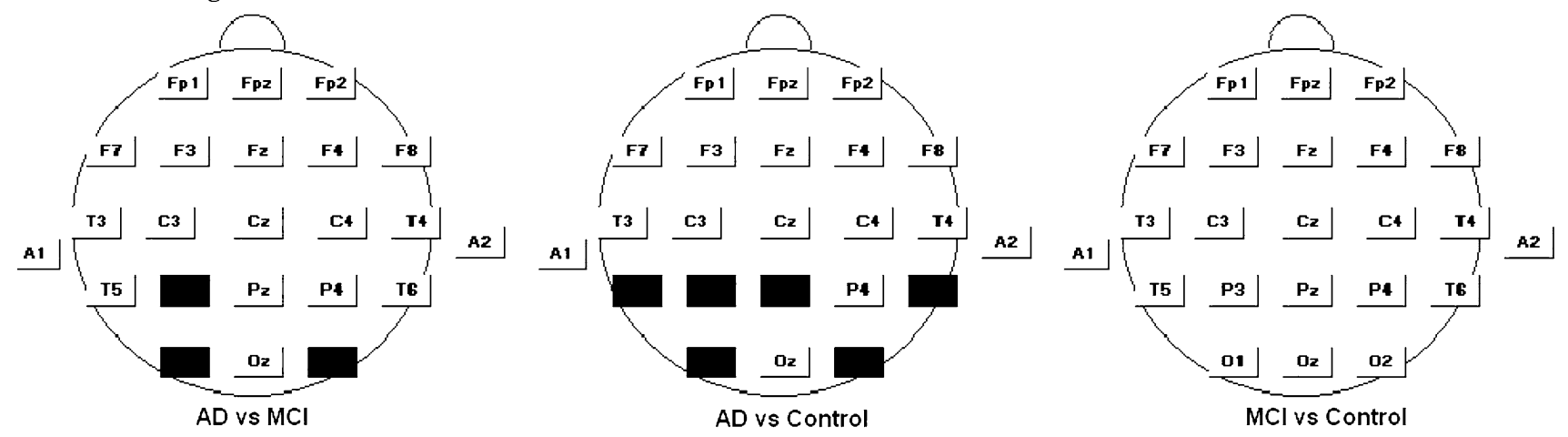

Story listening task
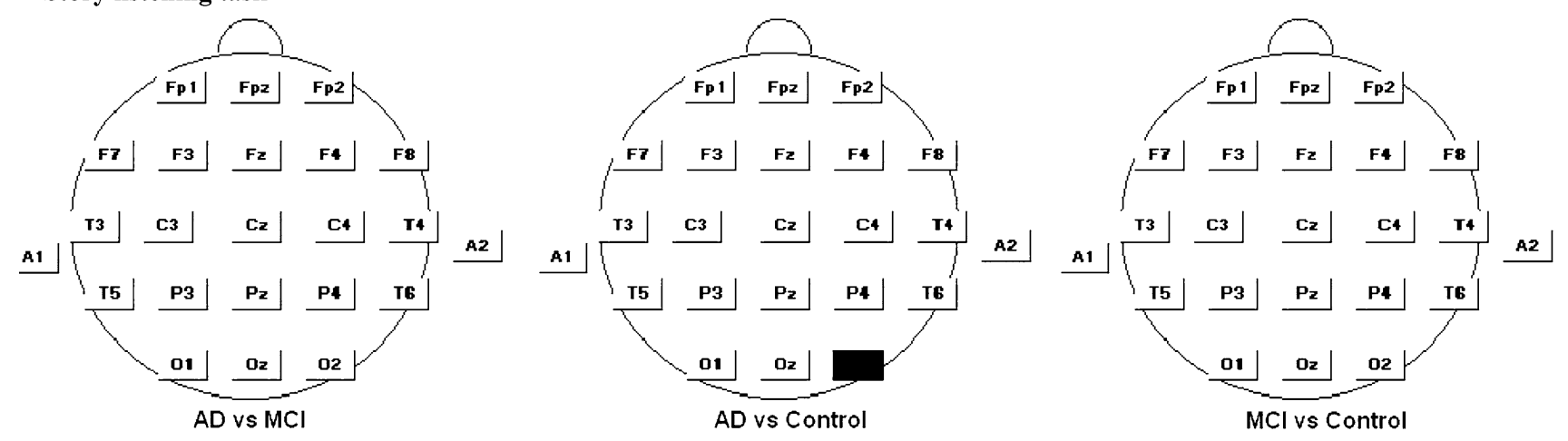

Visual task
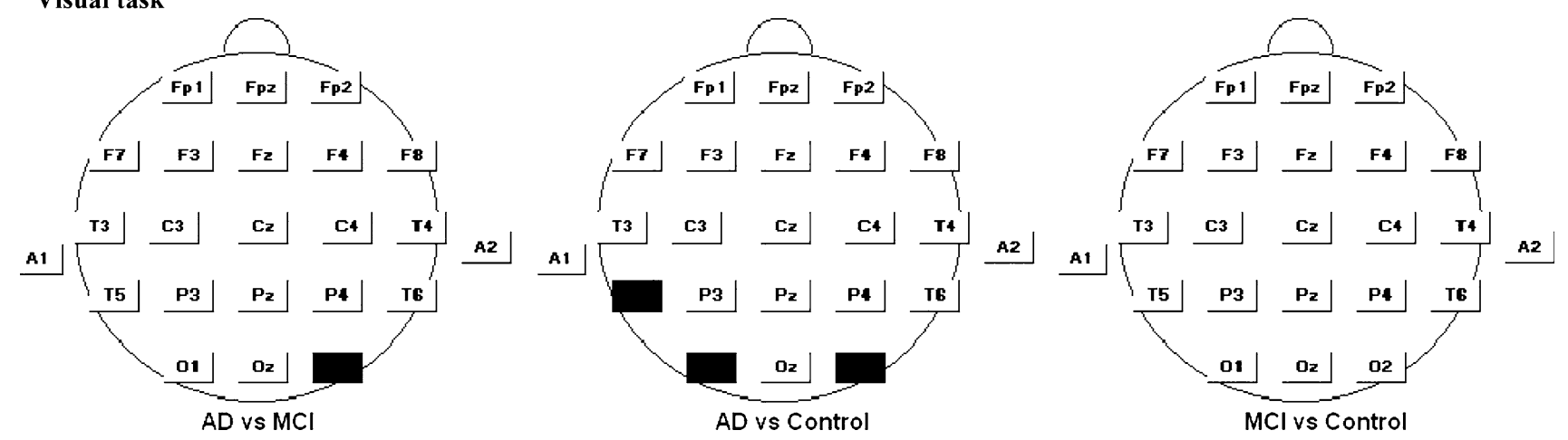

Fig. 1 Group comparison of GBP in different paradigms. Solid electrodes represent significantly increased GBP in AD compared to MCI, AD compared to control and MCI compared to control. All solid electrodes $(P<0.05)$ 
Table 2 Correlation between GBP and ADAS-cog

\begin{tabular}{lll}
\hline & Pearson's $R$ & Sig. $P$ \\
\hline Resting state & \\
C3 & 0.38 & 0.007 \\
Pz & 0.39 & 0.006 \\
P3 & 0.39 & 0.016 \\
P4 & 0.38 & 0.007 \\
T6 & 0.39 & 0.005 \\
O1 & 0.47 & 0.001 \\
O2 & 0.46 & 0.001 \\
Music listening & & \\
Pz & 0.38 & 0.005 \\
P3 & 0.37 & 0.007 \\
T5 & 0.38 & 0.005 \\
T6 & 0.28 & 0.005 \\
O1 & 0.46 & 0.001 \\
O2 & 0.47 & 0.001 \\
Story listening & & \\
O2 & 0.33 & 0.018 \\
Visual task & & \\
T5 & 0.34 & 0.011 \\
O1 & 0.37 & 0.006 \\
O2 & 0.41 & 0.002 \\
\hline Pearson's $R$ & & \\
& & \\
& &
\end{tabular}

Pearson's $R$ represents the correlation coefficient between GBP and ADAS-cog

The $P$-value represents the significance of the correlation

The story listening task showed increased GBP in the AD group for Fp1 $\left(F_{1,1}=4.8, P=0.044\right)$ and $\mathrm{F} 4$ $\left(F_{1,1}=4.7, P=0.049\right)$ compared to resting state. In the MCI group there was no significant increase or decrease of GBP during story listening compared to resting state. In the control group GBP increased during story listening at F3 $\left(F_{1,1}=5.2, P=0.035\right), \mathrm{P} 3\left(F_{1,1}=4.9, P=0.039\right)$, T5 $\left(F_{1,1}=10.7, P=0.004\right)$ and T6 $\left(F_{1,1}=7.8, P=0.007\right)$ compared to resting state. When the increase of GBP in story listening was analysed for all subjects irrespective of patient group a significant difference was shown for F3, F4, F5, F7, F8, Fp1, T5 andT6. The $F$ and $P$ values were in the same order of magnitude as when the groups were analysed separately. The percentage of increase in GBP due to story listening was $134 \%$ in the $\mathrm{AD}, 49 \%$ in $\mathrm{MCI}$ and $68 \%$ in controls. Statistical analysis of story listening related GBP increase showed no significant differences between the groups.

The visual task showed no increased GBP in the AD group compared to resting state. In the MCI group the visual task showed increased GBP at $\mathrm{O} 1 \quad\left(F_{1,1}=6.3\right.$, $P=0.016)$ compared to resting state. In the control group the visual task showed increased GBP at Fz $\left(F_{1,1}=4.4\right.$, $P=0.049)$ compared to resting state. When the increase of
GBP in the visual task was analysed for all subjects irrespective of patient group a significant difference was shown for F3, F4, F5, F7, F8, Fp1, T5 and T6. The $F$ and $P$ values were in the same order of magnitude as when the groups were analysed separately.

The percentage of increased GBP due to the visual task was $57 \%$ in $\mathrm{AD}, 26 \%$ in $\mathrm{MCI}$ and $62 \%$ in the control group. Statistical analysis of visual task related GBP increase showed no significant differences between the groups.

The differences between task performance and resting state was highest for the $\mathrm{AD}$ and control group. None of the electrodes showed decreased GBP in task performance compared to resting state condition.

\section{Effect of EMG on GBP}

The removal of EMG contaminated epochs resulted in a general decrease of GBP in all conditions (see Table 3). The original differences between the groups remained in the resting state and music listening conditions, except for the $\mathrm{O} 1$ and $\mathrm{O} 2$ electrodes. In the story listening task there were no effects on the differences between the groups. In the visual task the original differences were no longer significant after EMG correction. The contamination of EMG was found highest in the $\mathrm{O} 1$ and $\mathrm{O} 2$ electrodes.

\section{Discussion}

Contrary to our hypothesis we found a higher instead of a lower GBP in AD compared to MCI and controls.

A first step in our analysis was to ensure that differences in GBP between the groups are reproducible and stable, by evaluating test-retest reliability (TRR). An earlier study using a similar design demonstrated a satisfying TRR in young healthy subjects (Frund et al. 2007), but TRR has not been reported in studies with cognitively impaired patients. In the current study, out of 36 comparisons (i.e. three groups, three electrodes and four task conditions) made between the sessions with a paired samples $t$-test, only three significant differences between sessions were found; one difference in the resting state condition in the $\mathrm{AD}$ group, and two in the control group. In spite of these differences, the correlation between the two sessions was high for all electrodes. As shown in Table 1, the resting state GBP is higher in the first session compared to the second. This is most likely related to habituation or diminished arousal in the second session. Another point is that cognitive activity during resting state is an uncontrolled condition that is therefore more difficult to compare between subjects and sessions than under task conditions. The correlation between the sessions in the task conditions 


\section{Music listening task}
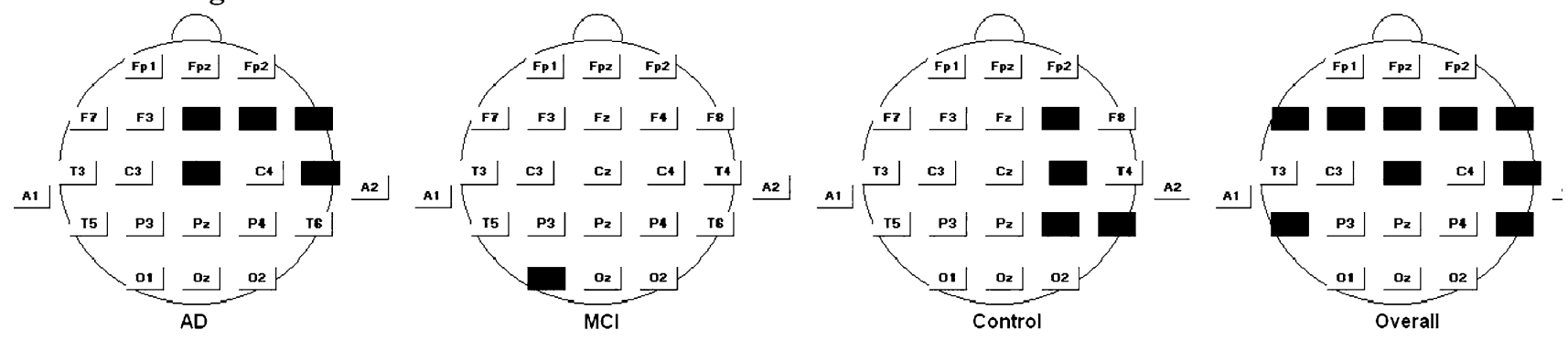

Story listening
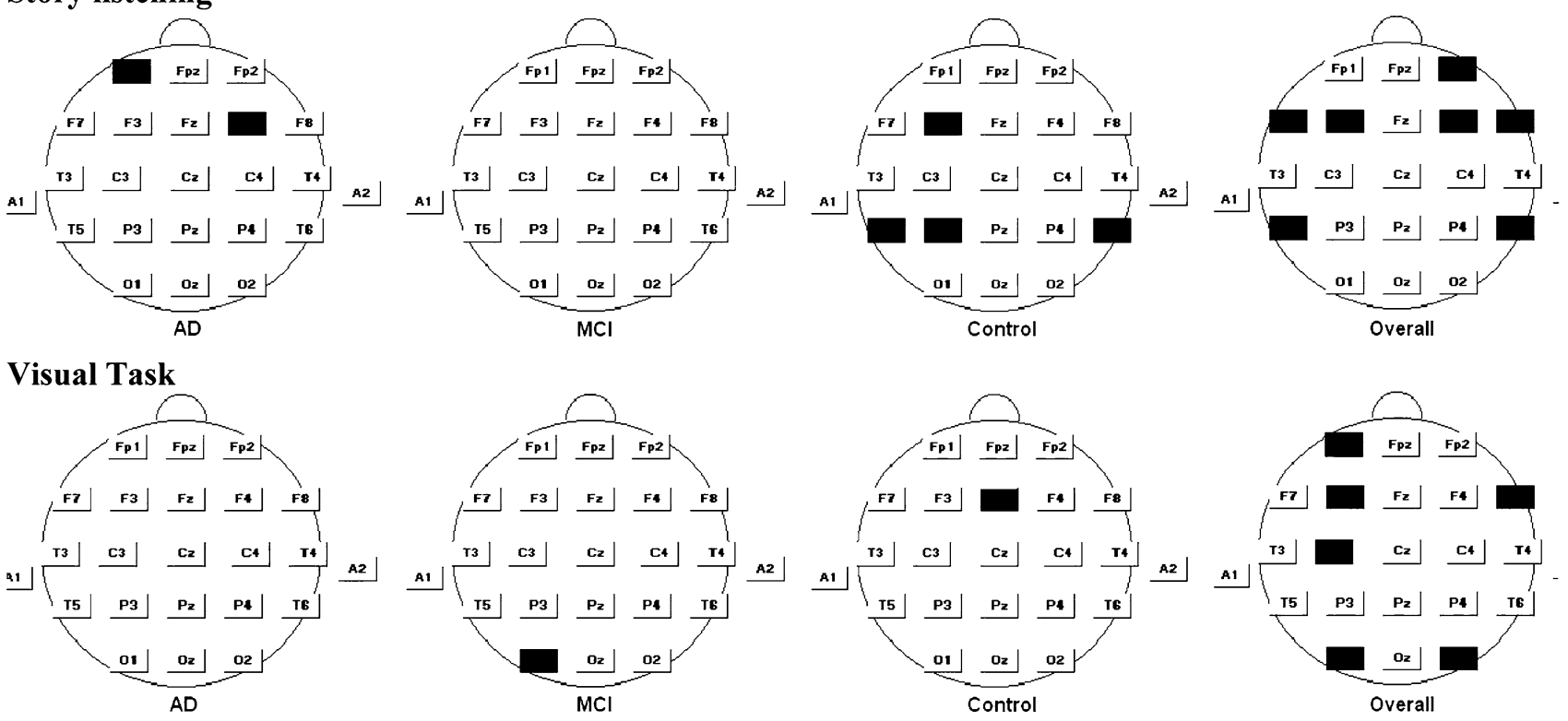

Fig. 2 The solid electrodes represent significantly increased GBP during task performance compared to resting state $(P<0.05)$. The results are presented for each group separately. Overall represents significantly increased GBP in task condition for all subjects irrespective of subject group

was high to very high for all paradigms, except for the visual task. For the latter no satisfying explanation could be found. Overall the TRR results suggest that GBP measures across all stimulus conditions are reproducible in healthy elderly subjects as well as in cognitively impaired patients.

The differences in GBP between the groups are located in the parietal, occipital and posterior temporal regions. These brain areas are also thought to be affected by typical $\mathrm{AD}$ neuropathological manifestations (i.e. neuritic plaques and neurofibrillary tangles) especially in the milder stages of AD (Braak and Braak 1995). The congruence in the location of the GBP changes and AD neuropathology suggests a specific association. Furthermore, we found a significant increase of GBP during task performance compared to resting state, similar to what was shown in healthy subjects by (Fitzgibbon et al. 2004) for all groups. The difference between the groups in task condition was most prominent in the music listening task, which might be related to the complexity of the music stimuli. Music might contain a broader auditory spectrum than the spoken text in story listening and could therefore require more attentional recourses. This increase of GBP during task performance compared to rest was higher in $\mathrm{AD}$ than the other groups in music listening and story listening. This task related difference was however only significant in T5 during music listening, which is probably related to lack of statistical power. The current results suggest that the difference in GBP between the groups was increased by task performance but this needs further substantiation by studies with larger sample sizes.

A difficulty with this type of studies is that gamma band and EMG share the same frequency. A recent study by Whitham et al. (2007) showed convincing evidence for major effects of EMG on GBP. Therefore we retrospectively tried to eliminate this factor. The post-hoc EMG analysis showed a general decrease of GBP in all conditions and for all electrodes. Nevertheless, it was shown that the major results of this study are sustained after reduction of the EMG contamination. The conservative procedure resulted in the removal of suspect EMG epochs, but also diminished the number of epochs with valid gamma band activity. It has to be noted that it is difficult to discriminate 
Table 3 Effect of EMG on GBP

\begin{tabular}{|c|c|c|c|}
\hline & Mean $\mu \mathrm{V}^{2} \mathrm{UC}$ & Mean $\mu \mathrm{V}^{2} \mathrm{C}$ & Sig. $P$ \\
\hline \multicolumn{4}{|c|}{ Resting state } \\
\hline $\mathrm{C} 3$ & 0.68 & 0.48 & 0.048 \\
\hline $\mathrm{Pz}$ & 0.45 & 0.42 & 0.027 \\
\hline $\mathrm{P} 3$ & 0.52 & 0.46 & 0.024 \\
\hline $\mathrm{P} 4$ & 0.44 & 0.40 & 0.031 \\
\hline T6 & 0.56 & 0.43 & 0.026 \\
\hline O1 & 1.03 & 0.60 & 0.018 \\
\hline $\mathrm{O} 2$ & 0.86 & 0.57 & 0.050 \\
\hline \multicolumn{4}{|c|}{ Music listening } \\
\hline $\mathrm{Pz}$ & 0.52 & 0.47 & 0.118 \\
\hline $\mathrm{P} 3$ & 0.59 & 0.52 & 0.085 \\
\hline T5 & 0.95 & 0.59 & 0.064 \\
\hline T6 & 0.76 & 0.48 & 0.002 \\
\hline $\mathrm{O} 1$ & 0.98 & 0.58 & 0.002 \\
\hline $\mathrm{O} 2$ & 0.86 & 0.54 & 0.005 \\
\hline \multicolumn{4}{|c|}{ Story listening } \\
\hline $\mathrm{O} 2$ & 0.85 & 0.68 & 0.000 \\
\hline \multicolumn{4}{|c|}{ Visual task } \\
\hline T5 & 0.83 & 0.76 & 0.085 \\
\hline O1 & 0.88 & 0.77 & 0.004 \\
\hline $\mathrm{O} 2$ & 0.75 & 0.75 & 0.000 \\
\hline
\end{tabular}

Paired samples $t$-test between corrected (C) and uncorrected (UC) EEG

The $P$-value represents the significance of the difference between corrected and uncorrected EEG

low voltage EMG from gamma band activity as they share the same frequency. To the best of our knowledge there are no automated algorithms that remove or reduce EMG from the EEG without having an effect on GBP. Therefore we considered the filtering and visual inspection as an adequate method to remove EMG artefacts. The study by Whitham et al. (2007) together with the current results suggest that in induced gamma band studies EMG activity should be taken into account.

A methodologically strong aspect of the current study is that only patients naïve for psychoactive drugs were included in this study. Almost all other studies on gamma band activity in AD included patients on cholinesterase inhibiting drugs, NMDA receptor antagonists or other psychoactive drugs. Several studies have reported that cholinesterase inhibitors have a strong impact on the EEG signal (Kogan et al. 2001; Rodriguez et al. 2002, 2004). This difference between the current study and previous studies might explain why elevated GBP has not been reported earlier. One other study showed enhanced GBP in psychoactive drug naïve $A D$ patients using a $40-\mathrm{Hz}$ steady state response (SSR) paradigm (Osipova et al. 2006).
The finding of increased GBP cannot be explained in a straightforward manner. In contrast to the current findings there are two that showed decreased GBP in $\mathrm{AD}$, one in humans and one in animals. The human study showed decreased GBP in a 40-Hz SSR paradigm in AD compared to controls (Ribary et al. 1991). In the animal study GBP was decreased in APP over-expressing mice, measured invitro (Driver et al. 2007). It has to be noted that the methods used in these two studies, fundamentally differ from induced GBP measurements and that therefore these results cannot be compared head to head. It would however for future research be interesting to study the relation between different type of gamma band paradigms in animals and in humans.

Although in the current study relatively simple tasks were used, attentional demands might play an important role in controlled processing during task performance. Increased attention might be required in $\mathrm{AD}$ as patients have more difficulty in processing information in an automatic manner compared to the MCI and control group. The enhanced GBP in AD might therefore reflect an increased level of controlled processing and effortful attention as a functional compensation mechanism in AD. Previous studies have shown that as attentional demand or task difficulty increases, GBP increases (Landau et al. 2007; Tallon-Baudry et al. 2005; Tiitinen et al. 1993). The hypothesis of different attentional demands between the groups is however not substantiated by the current data. In the resting state paradigm no GBP differences between the groups would be expected since there was no selective cognitive activity ongoing. Since the current study showed resting state differences, it cannot be concluded that enhanced GBP in $\mathrm{AD}$ is primarily the result of a difference in attentional demands. An alternative explanation might be that GBP is continuously enhanced in AD irrespective of sensory stimulation or specific demands on cognitive processing. Decreased resting state gamma band synchronization in $\mathrm{AD}$ was earlier demonstrated in the occipitoparietal regions (Koenig et al. 2005; Stam et al. 2002). An fMRI study showed that the posterior cingulate cortex and medial parietal cortex, areas usually activated in resting state condition, and deactivated in task condition are more active in $\mathrm{AD}$ subjects than in healthy controls during task performance (Lustig et al. 2003). In the current study GBP was increased in resting state in the same brain areas; the occipito-parietal regions. Another aspect is that induced gamma band oscillations, consist of task-related components as well as task-unrelated components. These taskunrelated oscillations are continuously present and are not lost during averaging of the data. This is in contrast to event-related designs where task-unrelated activity vanishes during pre-processing of the data. These taskunrelated oscillations therefore might have an effect on 
GBP and could be enhanced in AD. The latter point together with the fMRI data suggests that the so-called default mode is more active in AD and that this involves the gamma band. Increased default activity might be related to decreased cortical inhibition in AD. Decreased inhibition and hyper excitability of cortical responses in AD has been suggested by a number of studies (Di Lazzaro et al. 2004; Nardone et al. 2006a, b). Cortical disinhibition and hyper excitability are often associated with dysfunction of glutamate and GABA neurotransmission, which is a known characteristic of AD pathology (Francis 2005). The role of GABA and glutamate in the regulation of gamma band has been shown in vitro and suggest glutamate as a generator of gamma band oscillations and GABA as an inhibitor (Whittington et al. 2000). How these neurotransmitters regulate gamma band oscillations in the human brain and how this is reflected in scalp EEG is however not clear.

The present study shows a discrepancy with previous studies that showed decreased gamma band synchronization in AD (Koenig et al. 2005; Stam et al. 2002). This decrease in gamma band synchronization can be explained by the loss of long distance corticocortical connections that characterize AD. A study by Stam et al. (2006) showed impaired synchronization of the gamma band especially in the long distance connections. The results from the current study showing increased local GBP in AD suggest that the local networks are preserved. Increased local GBP might therefore reflect a compensation mechanism for decreased long distance connectivity. In the present study, synchronization was not calculated but the higher GBP might represent impaired information integration between different brain areas. Whether there is a relation between GBP and synchronization is dependent on the method used to calculate synchronization (Pereda et al. 2005). For future research it would be interesting to compare synchronization and power data in the same study sample.

In the current study we were not able to show a difference in GBP between the MCI and control group. Since ADAS-cog separated these two groups significantly it can be concluded that this is not the result of a small difference in cognitive status. The lack of a statistical difference may be related to the fact that MCI is an ambiguous construct of which the criteria include a heterogeneous population (Visser and Brodaty 2006). This might explain that there is no difference between the MCI and control groups. Nevertheless, was there a non-significant trend towards a difference between MCI and controls that was in line with our hypothesis that the GBP in the MCI group would be in between the GBP of the $\mathrm{AD}$ and control group.

\section{Conclusion}

Our results suggest that GBP is a reproducible measure that is able to differentiate $\mathrm{AD}$ patients from $\mathrm{MCI}$ and control subjects. Whether induced GBP measured during ongoing activity is a valid method needs to be substantiated by future research.

Acknowledgments This work was financially supported by research agreement CSMD Ref No: 002509 from GlaxoSmithKline, Cambridge, UK. We thank Lieke Smits, MSc for her help with the collection of the data.

Open Access This article is distributed under the terms of the Creative Commons Attribution Noncommercial License which permits any noncommercial use, distribution, and reproduction in any medium, provided the original author(s) and source are credited.

\section{References}

Basar E, Basar-Eroglu C, Karakas S, Schurmann M (2001) Gamma, alpha, delta, and theta oscillations govern cognitive processes. Int J Psychophysiol 39(2-3):241-248

Braak H, Braak A (1995) Staging of Alzheimer's disease-related neurofibrillary changes. Neurobiol Aging 16(3):271-284

Di Lazzaro V, Oliviero A, Pilato F, Saturno E, Dileone M, Marra C et al (2004) Motor cortex hyperexcitability to transcranial magnetic stimulation in Alzheimer's disease. J Neurol Neurosurg Psychiatry 75(4):555-559

Driver JE, Racca C, Cunningham MO, Towers SK, Davies CH, Whittington MA et al (2007) Impairment of hippocampal gamma-frequency oscillations in vitro in mice overexpressing human amyloid precursor protein (APP). Eur J NeuroSci 26(5):1280-1288

Fitzgibbon SP, Pope KJ, Mackenzie L, Clark CR, Willoughby JO (2004) Cognitive tasks augment gamma EEG power. Clin Neurophysiol 115(8):1802-1809

Francis PT (2005) The interplay of neurotransmitters in Alzheimer's disease. CNS Spectr 10(11 Suppl 18):6-9

Frund I, Schadow J, Busch NA, Korner U, Herrmann CS (2007) Evoked gamma oscillations in human scalp EEG are test-retest reliable. Clin Neurophysiol 118(1):221-227

Galambos R, Makeig S, Talmachoff PJ (1981) A 40-Hz auditory potential recorded from the human scalp. Proc Natl Acad Sci USA 78(4):2643-2647

Hachinski VC, Lassen NA, Marshall J (1974) Multi-infarct dementia. A cause of mental deterioration in the elderly. Lancet 2(7874):207-210

Huang C, Wahlund LO, Dierks T, Julin P, Winblad B, Jelic B (2000) Discrimination of Alzheimer's disease and mild cognitive impairment by equivalent EEG sources: a cross-sectional and longitudinal study. Clin Neurophysiol 111:1961-1967

Jelic V, Shigeta M, Julin P, Almkvist O, Winblad B, Wahlund LO (1996) Quantitative electroencephalography power and coherence in Alzheimer's disease and mild cognitive impairment. Dementia 7(6):314-323

Jeong J (2004) EEG dynamics in patients with Alzheimer's disease. Clin Neurophysiol 115(7):1490-1505

Kaiser J, Lutzenberger W (2003) Induced gamma-band activity and human brain function. Neuroscientist 9(6):475-484 
Kaiser J, Lutzenberger W (2005) Human gamma-band activity: a window to cognitive processing. NeuroReport 16(3):207-211

Koenig T, Prichep L, Dierks T, Hubl D, Wahlund LO, John ER et al (2005) Decreased EEG synchronization in Alzheimer's disease and mild cognitive impairment. Neurobiol Aging 26(2):165-171

Kogan EA, Korczyn AD, Virchovsky RG, Klimovizky S, Treves TA, Neufeld MY (2001) EEG changes during long-term treatment with donepezil in Alzheimer's disease patients. J Neural Transm 108(10):1167-1173

Landau AN, Esterman M, Robertson LC, Bentin S, Prinzmetal W (2007) Different effects of voluntary and involuntary attention on EEG activity in the gamma band. J Neurosci 27(44):1198611990

Lustig C, Snyder AZ, Bhakta M, O’Brien KC, McAvoy M, Raichle ME et al (2003) Functional deactivations: change with age and dementia of the Alzheimer type. Proc Natl Acad Sci USA 100(24):14504-14509

McKhann G, Drachman D, Folstein M, Katzman R, Price D, Stadlan EM (1984) Clinical diagnosis of Alzheimer's disease: report of the NINCDS-ADRDA Work Group under the auspices of Department of Health and Human Services Task Force on Alzheimer's Disease. Neurology 34(7):939-944

Nardone R, Bratti A, Tezzon F (2006a) Motor cortex inhibitory circuits in dementia with Lewy bodies and in Alzheimer's disease. J Neural Transm 113(11):1679-1684

Nardone R, Marth R, Ausserer H, Bratti A, Tezzon F (2006b) Reduced short latency afferent inhibition in patients with Down syndrome and Alzheimer-type dementia. Clin Neurophysiol 117(10):2204-2210

Osipova D, Pekkonen E, Ahveninen J (2006) Enhanced magnetic auditory steady-state response in early Alzheimer's disease. Clin Neurophysiol 117(9):1990-1995

Pereda E, Quiroga RQ, Bhattacharya J (2005) Nonlinear multivariate analysis of neurophysiological signals. Prog Neurobiol 77(12): $1-37$

Petersen RC, Doody R, Kurz A, Mohs RC, Morris JC, Rabins PV et al (2001) Current concepts in mild cognitive impairment. Arch Neurol 58(12):1985-1992

Ribary U, Ioannides AA, Singh KD, Hasson R, Bolton JP, Lado F et al (1991) Magnetic field tomography of coherent thalamocortical 40-Hz oscillations in humans. Proc Natl Acad Sci USA 88(24):11037-11041

Rodriguez G, Vitali P, De Leo C, De Carli F, Girtler N, Nobili F (2002) Quantitative EEG changes in Alzheimer patients during long-term donepezil therapy. Neuropsychobiology 46(1):49-56

Rodriguez R, Kallenbach U, Singer W, Munk MH (2004) Short- and long-term effects of cholinergic modulation on gamma oscillations and response synchronization in the visual cortex. J Neurosci 24(46):10369-10378

Rosen WG, Terry RD, Fuld PA, Katzman R, Peck A (1980) Pathological verification of ischemic score in differentiation of dementias. Ann Neurol 7(5):486-488
Rosen WG, Mohs RC, Davis KL (1984) A new rating scale for Alzheimer's disease. Am J Psychiatry 141(11):1356-1364

Schmand B, Bakker D, Saan R, Louman J (1991) The Dutch reading test for adults: a measure of premorbid intelligence level. Tijdschr Gerontol Geriatr 22(1):15-19

Singer W, Gray CM (1995) Visual feature integration and the temporal correlation hypothesis. Annu Rev Neurosci 18:555-586

Stam CJ, van Walsum AM, Pijnenburg YA, Berendse HW, de Munck JC, Scheltens P et al (2002) Generalized synchronization of MEG recordings in Alzheimer's Disease: evidence for involvement of the gamma band. J Clin Neurophysiol 19(6):562-574

Stam CJ, van der Made Y, Pijnenburg YA, Scheltens P (2003) EEG synchronization in mild cognitive impairment and Alzheimer's disease. Acta Neurol Scand 108(2):90-96

Stam CJ, Jones BF, Manshanden I, van Walsum AM, Montez T, Verbunt JP et al (2006) Magnetoencephalographic evaluation of resting-state functional connectivity in Alzheimer's disease. Neuroimage 32(3):1335-1344

Tallon-Baudry C, Bertrand O (1999) Oscillatory gamma activity in humans and its role in object representation. Trends Cogn Sci 3(4):151-162

Tallon-Baudry C, Bertrand O, Henaff MA, Isnard J, Fischer C (2005) Attention modulates gamma-band oscillations differently in the human lateral occipital cortex and fusiform gyrus. Cereb Cortex 15(5):654-662

Tiitinen H, Sinkkonen J, Reinikainen K, Alho K, Lavikainen J, Naatanen R (1993) Selective attention enhances the auditory 40$\mathrm{Hz}$ transient response in humans. Nature 364(6432):59-60

van Boxtel MP, Buntinx F, Houx PJ, Metsemakers JF, Knottnerus A, Jolles J (1998) The relation between morbidity and cognitive performance in a normal aging population. J Gerontol A Biol Sci Med Sci 53(2):M147-M154

Verhey FR, Houx P, Van Lang N, Huppert F, Stoppe G, Saerens J et al (2004) Cross-national comparison and validation of the Alzheimer's Disease Assessment Scale: results from the European Harmonization Project for Instruments in Dementia (EURO-HARPID). Int J Geriatr Psychiatry 19(1):41-50

Visser PJ, Brodaty H (2006) MCI is not a clinically useful concept. Int Psychogeriatr 18(3):402-409; discussion 409-414

Whittington MA, Traub RD, Kopell N, Ermentrout B, Buhl EH (2000) Inhibition-based rhythms: experimental and mathematical observations on network dynamics. Int $\mathrm{J}$ Psychophysiol 38(3):315-336

Whitham EM, Pope KJ, Fitzgibbon SP, Lewis T, Clark CR, Loveless $S$ et al (2007) Scalp electrical recording during paralysis: quantitative evidence that EEG frequencies above $20 \mathrm{~Hz}$ are contaminated by EMG. Clin Neurophysiol 118(8):1877-1888 